\title{
Article \\ Trimethoxycinnamates and Their Cholinesterase Inhibitory Activity
}

\author{
Jiri Kos ${ }^{1, *}$, Tomas Strharsky ${ }^{1,2}$, Sarka Stepankova ${ }^{3} \mathbb{D}$, Katarina Svrckova ${ }^{3} \mathbb{D}_{\text {, Michal Oravec }}^{4}$, Jan Hosek ${ }^{5} \mathbb{D}$, \\ Ales Imramovsky ${ }^{6}$ and Josef Jampilek ${ }^{1,7}$ (D)
}

1 Regional Centre of Advanced Technologies and Materials, Czech Advanced Technology and Research Institute, Palacky University, Slechtitelu 27, 78371 Olomouc, Czech Republic; strharsky.t@gmail.com (T.S.); josef.jampilek@gmail.com (J.J.)

2 Department of Physical Chemistry, Faculty of Science, Palacky University, 17, Listopadu 12, 77146 Olomouc, Czech Republic

3 Department of Biological and Biochemical Sciences, Faculty of Chemical Technology, University of Pardubice, Studentska 573, 53210 Pardubice, Czech Republic; sarka.stepankova@upce.cz (S.S.);

katarina.svrckova@upce.cz (K.S.)

4 Global Change Research Institute CAS, Belidla 986/4a, 60300 Brno, Czech Republic; oravec.m@czechglobe.cz

5 Department of Pharmacology and Toxicology, Veterinary Research Institute, Hudcova 296/70, 62100 Brno, Czech Republic; hosek.jan@vri.cz

6 Institute of Organic Chemistry and Technology, Faculty of Chemical Technology, University of Pardubice, Studentska 573, 53210 Pardubice, Czech Republic; ales.imramovsky@upce.cz

7 Institute of Neuroimmunology, Slovak Academy of Sciences, Dubravska Cesta 9, 84510 Bratislava, Slovakia

* Correspondence: jiri.kos@upol.cz

check for updates

Citation: Kos, J.; Strharsky, T.; Stepankova, S.; Svrckova, K.; Oravec, M.; Hosek, J.; Imramovsky, A.; Jampilek, J. Trimethoxycinnamates and Their Cholinesterase Inhibitory Activity. Appl. Sci. 2021, 11, 4691. https://doi.org/10.3390/ app11104691

Academic Editor: Antony

C. Calokerinos

Received: 31 March 2021

Accepted: 17 May 2021

Published: 20 May 2021

Publisher's Note: MDPI stays neutral with regard to jurisdictional claims in published maps and institutional affiliations.

Copyright: (c) 2021 by the authors. Licensee MDPI, Basel, Switzerland. This article is an open access article distributed under the terms and conditions of the Creative Commons Attribution (CC BY) license (https:// creativecommons.org/licenses/by/ $4.0 /)$.
Abstract: A series of twelve nature-inspired 3,4,5-trimethoxycinnamates were prepared and characterized. All compounds, including the starting 3,4,5-trimethoxycinnamic acid, were tested for their ability to inhibit acetylcholinesterase (AChE) and butyrylcholinesterase (BChE) in vitro; the selectivity index (SI) was also determined. 2-Fluororophenyl (2E)-3-(3,4,5-trimethoxyphenyl)-prop-2enoate demonstrated the highest SI (1.71) in favor of BChE inhibition. 2-Chlorophenyl (2E)-3-(3,4,5trimethoxyphenyl)prop-2-enoate showed the highest $\mathrm{AChE-inhibiting}\left(\mathrm{IC}_{50}=46.18 \mu \mathrm{M}\right)$ as well as BChE-inhibiting $\left(\mathrm{IC}_{50}=32.46 \mu \mathrm{M}\right)$ activity with an SI of 1.42. The mechanism of action of the most potent compound was determined by the Lineweaver-Burk plot as a mixed type of inhibition. An in vitro cell viability assay confirmed the insignificant cytotoxicity of the discussed compounds on the two cell lines. Trends between structure, physicochemical properties and activity were discussed.

Keywords: trimethoxycinnamates; cholinesterase-inhibiting activity; cytotoxicity; structure-activity relationships

\section{Introduction}

Nature is an inspirational and rich source of compounds with biological effects. More than a half of all small-molecule-approved drugs between 1981 and 2014 belong to groups of natural products or mimic natural products [1]. Many plant secondary metabolites [2] have also been used as model compounds for the subsequent development of synthetic drugs [3-6]. One such example is a cinnamic acid and its derivatives, e.g., coumaric acids, caffeic acid, ferulic acid or sinapic acid, which are widely distributed in many plants and assure their many physiological processes. They also serve as precursors for other compounds in plants such as lignols, (iso)flavonoids, coumarins, aurones, stilbenes, catechin, phenylpropanoids, etc. [7].

Natural cinnamic acid also serves as a precursor for new synthetic compounds. Synthetic derivatives of cinnamic acid showed interesting antimicrobial, anticancer, antiparasitic, neurological, antioxidant, and anti-inflammatory activities in vitro [8]. 3,4,5Trimethoxycinnamic acid (TMCA) is an ingredient isolated from the root of Polygala tenuifolia Willd. (Polygalaceae), and its extracts have been used as traditional sedative drugs in 
China, Japan and Korea [9-11]. TMCA has been reported to have antidepressant, anticonvulsant and sedative activity $[9,12,13]$. In recent years, a large number of TMCA derivatives with various biological effects have been synthesized, such as antinarcotic [14], antitumor [15-18], antiviral [19,20], anticonvulsant [21] and anti-inflammatory [21,22] agents. Published articles indicate that TMCA and its ester and amide analogues exhibit central nervous system activity, including antinarcotic, neuroprotective, anti-Alzheimer's and anticonvulsant effects. It involves several targets, such as 5-hydroxytryptamine receptor, prostaglandin $\mathrm{E}_{2}$ receptor 2 and nuclear factor erythroid 2-related factor 2, $\beta$-amyloid peptide (1-42), acetylcholinesterase (AChE), and butyrylcholinesterase (BChE) [14,22-25].

Alzheimer's disease (AD) accounts for $60 \%$ to $80 \%$ of dementia cases and is becoming an increasingly common disease not only in Western countries but also in low- and middleincome countries. It is estimated that more than 50 million people worldwide suffered from dementia by 2020, and that number is expected to rise to 82 million in 2030 and 152 million in 2050. For example, by the middle of the century, the estimated number of Americans aged 65 and over with $\mathrm{AD}$ could rise to 13.8 million, a sharp increase from the estimated 5.8 million Americans aged 65 and over who suffer from AD today. The latest available statistics from 2018 show that AD was the sixth most common cause of death in the United States [26,27]. The exact cause of Alzheimer's disease is still unclear. It is seemingly a multifactorial disease, which is also caused by stress, unfavorable environment, eating habits, family predisposition, etc. Several hypotheses are known, the most elaborate of which concern amyloid, tau protein and acetylcholine concentration. This cholinergic hypothesis is the oldest and has attracted the most attention [26-28]. Drugs used in clinical practice to alleviate $\mathrm{AD}$ modulate acetylcholine levels in the brain by inhibiting $\mathrm{AChE}$ [28-33]. In the early stages of AD, AChE level increases much faster than the level of BChE. On the other hand, as AD progresses, AChE level decreases in the brain, but $\mathrm{BChE}$ level remains unchanged or even increased (e.g., in the hippocampus and temporal cortex). Moreover, in the later stages of $\mathrm{AD}, \mathrm{BChE}$ replaces the function of $\mathrm{AChE}$ (i.e., hydrolysis of $\mathrm{ACh}$ ) [34,35]. Thus, inhibition of $\mathrm{BChE}$ in addition to $\mathrm{AChE}$ may provide therapeutic benefit not only in $\mathrm{AD}$. In addition, selective $\mathrm{BChE}$ inhibitors do not exhibit the adverse cholinergic effects that are characteristic of AChE inhibitors. Although there are several clinically used drugs, in terms of treatment quality, it is advisable to look for new AChE/BChE inhibitors with high activity, low side effects and low toxicity $[28,29,36-42]$.

In the last few years, cinnamic acid derivatives have been studied for their antiinvasive and anti-inflammatory activity [43-47]. However, a series of TMCA esters were prepared in this study and their anticholinesterase activity was investigated.

\section{Materials and Methods}

\subsection{General Methods}

All reagents were purchased from Merck (Sigma-Aldrich, St. Louis, MO, USA) and Alfa (Alfa-Aesar, Ward Hill, MA, USA). The melting points were determined on a Stuart SMP10 apparatus (Stone, UK) and are uncorrected. Infrared (IR) spectra were recorded on a Nicolet iS5 IR spectrometer (Thermo Scientific, West Palm Beach, FL, USA). The spectra were obtained by the accumulation of 256 scans with $2 \mathrm{~cm}^{-1}$ resolution in the region of $4000-450 \mathrm{~cm}^{-1}$. All ${ }^{1} \mathrm{H}$ - and ${ }^{13} \mathrm{C}-\mathrm{NMR}$ spectra were recorded on a JEOL JNM-ECA 600II device (600 MHz for ${ }^{1} \mathrm{H}$ and $150 \mathrm{MHz}$ for ${ }^{13} \mathrm{C}$, JEOL, Tokyo, Japan) in dimethyl sulfoxide- $d_{6}$ (DMSO- $\left.d_{6}\right) .{ }^{1} \mathrm{H}$ and ${ }^{13} \mathrm{C}$ chemical shifts $(\delta)$ are reported in ppm. High-resolution mass spectra were measured using a high-performance liquid chromatograph, Dionex UltiMate ${ }^{\circledR}$ 3000 (Thermo Scientific, West Palm Beach, FL, USA), coupled with an LTQ Orbitrap XL TM Hybrid Ion Trap-Orbitrap Fourier Transform Mass Spectrometer (Thermo Scientific) equipped with a HESI II (heated electrospray ionization) source in the positive mode. 


\subsection{Synthesis}

General Procedure Used to Synthesize Esters 1-12

Trimethoxycinnamic acid $(0.5 \mathrm{~g}, 0.2 \mathrm{mmol})$ was suspended in dry toluene $(5 \mathrm{~mL})$ at room temperature and thionyl chloride $(0.25 \mathrm{mmol})$ was added dropwise. The reaction mixture was stirred for $1 \mathrm{~h}$ under reflux. The residue was used directly in the next step. Into the solution of trimethoxycinnamic acid chloride in dry toluene $(5 \mathrm{~mL})$, triethylamine $(0.25 \mathrm{mmol})$ and corresponding substituted alcohol $(0.2 \mathrm{mmol})$ were added dropwise. The mixture was stirred under reflux for $1 \mathrm{~h}$, after which the solvent was removed under reduced pressure. The residue was extracted with EtOAc. Combined organic layers were washed with $1 \mathrm{M} \mathrm{HCl}$ and distilled water and dried over anhydrous $\mathrm{MgSO}_{4}$. The solvent was evaporated to dryness under reduced pressure. The crude product was recrystallized from $\mathrm{EtOH}$.

Phenyl (2E)-3-(3,4,5-trimethoxyphenyl)prop-2-enoate (1) [14]. Yield 56\%; Mp 105-107 ${ }^{\circ} \mathrm{C}$; IR (ATR, $\mathrm{cm}^{-1}$ ): 3041, 3012, 2939, 2835, 1721, 1631, 1580, 1507, 1495, 1485, 1468, 1455, 1437, 1416, 1337, 1321, 1266, 1241, 1180, 1156, 1122, 1067, 1023, 999, 971, 931, 903, 868, 846, 833, 816, 785, 755, 724, 687, 940, 603, 553; ${ }^{1} \mathrm{H}-\mathrm{NMR}$ (DMSO- $\left.d_{6}\right), \delta: 7.82(\mathrm{~d}, J=15.8 \mathrm{~Hz}, 1 \mathrm{H})$, 7.47-7.44 (m, 2H), 7.30-7.27 (m, 1H), 7.22-7.20 (m, 2H), $7.18(\mathrm{~s}, 2 \mathrm{H}), 6.92(\mathrm{~d}, J=15.8 \mathrm{~Hz}$, $1 \mathrm{H}), 3.84(\mathrm{~s}, 6 \mathrm{H}), 3.72(\mathrm{~s}, 3 \mathrm{H}) ;{ }^{13} \mathrm{C}-\mathrm{NMR}$ (DMSO-d $\left.d_{6}\right), \delta: 165.1,153.1,150.5,146.7,139.8,129.5$, 129.5, 125.8, 121.8, 116.4, 106.3, 60.1, 56.1; HR-MS: $\mathrm{C}_{18} \mathrm{H}_{19} \mathrm{O}_{5}[\mathrm{M}+\mathrm{H}]^{+}$calculated 315.1227 $\mathrm{m} / \mathrm{z}$, found $315.1228 \mathrm{~m} / \mathrm{z}$.

4-Methoxyphenyl (2E)-3-(3,4,5-trimethoxyphenyl)prop-2-enoate (2). Yield 75\%; Mp 131-133 ${ }^{\circ} \mathrm{C}$; IR (ATR, $\mathrm{cm}^{-1}$ ) 3000, 2967, 2945, 2842, 1703, 1627, 1582, 1502, 1453, 1419, 1339, 1321, 1277, 1246, 1186, 1155, 1121, 1099, 1020, 1008, 992, 941, 925, 915, 883, 863, 840, 832, 818, 805, 792, 779, 701, 658, 640, 629, 607, 544, 521, 487, 447; ${ }^{1} \mathrm{H}-\mathrm{NMR}$ (DMSO- $\left.d_{6}\right), \delta: 7.78(\mathrm{~d}, J=15.8 \mathrm{~Hz}, 1 \mathrm{H}), 7.17(\mathrm{~s}$, 2H), 7.14-7.11 (m, 2H), 6.99-6.96 (m, 2H), $6.89(\mathrm{~d}, J=15.8 \mathrm{~Hz}, 1 \mathrm{H}), 3.83(\mathrm{~s}, 6 \mathrm{H}), 3.77(\mathrm{~s}, 3 \mathrm{H}), 3.71$ $(\mathrm{s}, 3 \mathrm{H}) ;{ }^{13} \mathrm{C}-\mathrm{NMR}$ (DMSO- $\left.d_{6}\right), \delta: 165.4,156.8,153.1,146.4,143.9,139 ., 7,129.5,122.6,116.5,114.4$, 106.2, 60.1, 56.1, 55.4; HR-MS: $\mathrm{C}_{19} \mathrm{H}_{21} \mathrm{O}_{6}[\mathrm{M}+\mathrm{H}]^{+}$calculated $345.1333 \mathrm{~m} / \mathrm{z}$, found $345.1332 \mathrm{~m} / \mathrm{z}$.

4-Methylphenyl (2E)-3-(3,4,5-trimethoxyphenyl)prop-2-enoate (3). Yield 51\%; Mp 103-105 ${ }^{\circ} \mathrm{C}$; IR (ATR, $\mathrm{cm}^{-1}$ ): 2937, 2837, 1719, 1642, 1581, 1506, 1469, 1454, 1437, 1416, 1382, 1337, 1321, 1273, $1241,1187,1166,1156,1124,1021,998,972,939,925,917,861,829,817,808,788,774,706,632$, 603, 528, 517, 501; ${ }^{1} \mathrm{H}-\mathrm{NMR}$ (DMSO- $\left.d_{6}\right), \delta: 7.79(\mathrm{~d}, J=15.8 \mathrm{~Hz}, 1 \mathrm{H}), 7.25-7.23(\mathrm{~m}, 2 \mathrm{H}), 7.17(\mathrm{~s}$, 2H), 7.09-7.06 (m, 2H), $6.90(\mathrm{~d}, J=15.8 \mathrm{~Hz}, 1 \mathrm{H}), 3.83(\mathrm{~s}, 6 \mathrm{H}), 3.71(\mathrm{~s}, 3 \mathrm{H}), 3.32(\mathrm{~s}, 3 \mathrm{H}) ;{ }^{13} \mathrm{C}-\mathrm{NMR}$ (DMSO-d $d_{6}$ ), $\delta: 165.2,153.1,148.3,146.5,139.8,134.9,129.9,129.5,121.5,116.5,106.3,60.1,56.1$, 20.4; HR-MS: $\mathrm{C}_{19} \mathrm{H}_{21} \mathrm{O}_{5}[\mathrm{M}+\mathrm{H}]^{+}$calculated $329.1384 \mathrm{~m} / \mathrm{z}$, found $329.1380 \mathrm{~m} / \mathrm{z}$.

4-Fluorophenyl (2E)-3-(3,4,5-trimethoxyphenyl)prop-2-enoate (4). Yield 52\%; Mp 117-119 ${ }^{\circ} \mathrm{C}$; IR (ATR, $\mathrm{cm}^{-1}$ ): 3068, 3008, 2971, 2944, 2846, 1727, 1711, 1635, 1581, 1501, 1471, 1448, 1419, 1338, 1322, 1274, 1237, 1224, 1183, 1149, 1120, 1092, 1042, 1012, 999, 986, 943, 926, 848, 834, 824, 797, $785,761,746,707,657,635,520,604,529,519,458 ;{ }^{1} \mathrm{H}-\mathrm{NMR}$ (DMSO- $\left.d_{6}\right), \delta: 7.80(\mathrm{~d}, J=15.8 \mathrm{~Hz}$, 1H), 7.30-7.25 (m, 4H) $7.18(\mathrm{~s}, 2 \mathrm{H}), 6.91(\mathrm{~d}, J=15.8 \mathrm{~Hz}, 1 \mathrm{H}), 3.84(\mathrm{~s}, 6 \mathrm{H}), 3.71(\mathrm{~s}, 3 \mathrm{H}) ;{ }^{13} \mathrm{C}-\mathrm{NMR}$ (DMSO- $\left.d_{6}\right), \delta: 165.1,159.6(\mathrm{~d}, J=241.3 \mathrm{~Hz}), 153.1,146.9,146.6(\mathrm{~d}, J=2.9 \mathrm{~Hz}), 139.9,129.4$, $123.6(\mathrm{~d}, J=8.7 \mathrm{~Hz}), 116.1(\mathrm{~d}, J=4.3 \mathrm{~Hz}), 116.0,106.3,60.1,56.1$; HR-MS: $\mathrm{C}_{18} \mathrm{H}_{18} \mathrm{FO}_{5}[\mathrm{M}+\mathrm{H}]^{+}$ calculated $333.1133 \mathrm{~m} / \mathrm{z}$, found $333.1133 \mathrm{~m} / \mathrm{z}$.

4-Bromophenyl (2E)-3-(3,4,5-trimethoxyphenyl)prop-2-enoate (5). Yield 45\%; Mp 112-114 ${ }^{\circ} \mathrm{C}$; IR (ATR, $\mathrm{cm}^{-1}$ ) 3090, 3063, 3005, 2970, 2944, 2837, 1710, 1629, 1581, 1505, 1483, 1462, 1451, 1433, 1419, 1340, 1322, 1271, 1245, 1188, 1155, 1123, 1065, 1042, 1009, 999, 985, 945, 924, 916, 876, $858,846,839,827,821,802,782,765,751,715,673,656,644,628,601,584,523,499 ;{ }^{1} \mathrm{H}-\mathrm{NMR}$ (DMSO- $\left.d_{6}\right), \delta: 7.81(\mathrm{~d}, J=15.8 \mathrm{~Hz}, 1 \mathrm{H}), 7.65-7.62(\mathrm{~m}, 2 \mathrm{H}), 7.22-7.20(\mathrm{~m}, 2 \mathrm{H}), 7.18(\mathrm{~s}, 2 \mathrm{H}), 6.91$ $(\mathrm{d}, J=15.8 \mathrm{~Hz}, 1 \mathrm{H}), 3.83(\mathrm{~s}, 6 \mathrm{H}), 3.71(\mathrm{~s}, 3 \mathrm{H}) ;{ }^{13} \mathrm{C}-\mathrm{NMR}\left(\mathrm{DMSO}-d_{6}\right), \delta: 164.8,153.1,149.8,147.1$, 139.9, 132.4, 129.4, 124.2, 118.1, 116.1, 106.3, 60.1, 56.1; HR-MS: $\mathrm{C}_{18} \mathrm{H}_{18} \mathrm{BrO}_{5}[\mathrm{M}+\mathrm{H}]^{+}$calculated $393.0332 \mathrm{~m} / \mathrm{z}$, found $393.0323 \mathrm{~m} / \mathrm{z}$. 
2-Fluorophenyl (2E)-3-(3,4,5-trimethoxyphenyl)prop-2-enoate (6). Yield 60\%; Mp 165-167 ${ }^{\circ} \mathrm{C}$; IR (ATR, $\mathrm{cm}^{-1}$ ): 3044, 3010, 2963, 2942, 2837, 1717, 1628, 1580, 1508, 1498, 1466, 1454, 1437, 1419, $1337,1322,1273,1244,1225,1190,1176,1168,1156,1122,1097,1030,998,975,951,925,916,873$, $849,834,827,800,785,756,226,702,640,604,574,552,543,528,491,469 ;{ }^{1} \mathrm{H}-\mathrm{NMR}$ (DMSO- $\left.d_{6}\right), \delta$ : $7.85(\mathrm{~d}, J=16.5 \mathrm{~Hz}, 1 \mathrm{H}), 7.42-7.33(\mathrm{~m}, 3 \mathrm{H}), 7.28(\mathrm{td}, J=7.6 \mathrm{~Hz}, J=1.4 \mathrm{~Hz}, 1 \mathrm{H}), 7.20(\mathrm{~s}, 2 \mathrm{H}), 6.97$ $(\mathrm{d}, J=15.8 \mathrm{~Hz}, 1 \mathrm{H}), 3.84(\mathrm{~s}, 6 \mathrm{H}), 3.72(\mathrm{~s}, 3 \mathrm{H}) ;{ }^{13} \mathrm{C}-\mathrm{NMR}\left(\mathrm{DMSO}-d_{6}\right), \delta: 164.4,153.9(\mathrm{~d}, J=245.7$ $\mathrm{Hz}), 153.4,147.9,140.2,137.9(\mathrm{~d}, J=13.0 \mathrm{~Hz}), 129.5,127.8(\mathrm{~d}, J=7.2 \mathrm{~Hz}), 125.4(\mathrm{~d}, J=4.3 \mathrm{~Hz})$, $124.5,116.9$ (d, $J=18.8 \mathrm{~Hz}), 115.5,106.6,60.4,56.32$; HR-MS: $\mathrm{C}_{18} \mathrm{H}_{18} \mathrm{FO}_{5}[\mathrm{M}+\mathrm{H}]^{+}$calculated $333.1133 \mathrm{~m} / \mathrm{z}$, found $333.1129 \mathrm{~m} / \mathrm{z}$.

2-Chlorophenyl (2E)-3-(3,4,5-trimethoxyphenyl)prop-2-enoate (7). Yield 59\%; Mp 104-107 ${ }^{\circ} \mathrm{C}$; IR (ATR, $\mathrm{cm}^{-1}$ ): 2999, 2965, 2942, 2837, 1720, 1630, 1578, 1506, 1476, 1466, 1449, 1437, 1415, 1335, 1319, 1298, 1273, 1240, 1198, 1187, 1148, 1123, 1055, 1029, 996, 971, 951, 923, 914, 872, 841, 829, 816, 787, 762, 750, 739, 715, 683, 661, 637, 602, 563, 527, 796, 450; ${ }^{1} \mathrm{H}-\mathrm{NMR}$ (DMSO- $\left.\mathrm{d}_{6}\right), \delta: 7.86$ (d, $J=15.8 \mathrm{~Hz}, 1 \mathrm{H}), 7.61(\mathrm{dd}, J=8.2 \mathrm{~Hz}, J=1.4 \mathrm{~Hz}, 1 \mathrm{H}), 7.44(\mathrm{td}, J=7.6 \mathrm{~Hz}, J=1.4 \mathrm{~Hz}, 1 \mathrm{H}), 7.39$ (dd, $J=8.2 \mathrm{~Hz}, J=2.1 \mathrm{~Hz}, 1 \mathrm{H}), 7.34(\mathrm{td}, J=7.6 \mathrm{~Hz}, J=1.4 \mathrm{~Hz}, 1 \mathrm{H}), 7.21(\mathrm{~s}, 2 \mathrm{H}), 6.99(\mathrm{~d}, J=15.8 \mathrm{~Hz}$,

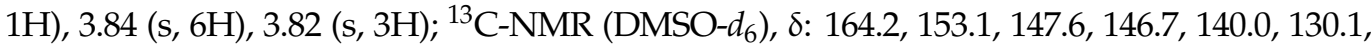
$129.3,128.5,127.6,126.0,124.4,115.5,106.5,60.1,56.1$; $\mathrm{HR}-\mathrm{MS}: \mathrm{C}_{18} \mathrm{H}_{18} \mathrm{ClO}_{5}[\mathrm{M}+\mathrm{H}]^{+}$calculated $349.0837 \mathrm{~m} / \mathrm{z}$, found $349.0837 \mathrm{~m} / \mathrm{z}$.

2,6-Dimethoxyphenyl (2E)-3-(3,4,5-trimethoxyphenyl)prop-2-enoate (8). Yield 69\%; Mp 155$157^{\circ} \mathrm{C}$; IR (ATR, $\mathrm{cm}^{-1}$ ): 3008, 2942, 2838, 1720, 1631, 1606, 1582, 1505, 1495, 1480, 1454, 1444, 1432, 1418, 1340, 1299, 1272, 1258, 1244, 1193, 1174, 1138, 1108, 1028, 1004, 982, 927, 866, $842,822,812,773,755,714,637,629,602,554,525,507,459 ;{ }^{1} \mathrm{H}-\mathrm{NMR}\left(\mathrm{DMSO}-d_{6}\right), \delta: 7.75(\mathrm{~d}$, $J=16.5 \mathrm{~Hz}, 1 \mathrm{H}), 7.19(\mathrm{t}, J=8.2 \mathrm{~Hz}, 1 \mathrm{H}), 7.18(\mathrm{~s}, 2 \mathrm{H}), 6.92(\mathrm{~d}, J=15.8 \mathrm{~Hz}, 1 \mathrm{H}), 6.77(\mathrm{~d}, J=8.2$ $\mathrm{Hz}, 2 \mathrm{H}), 3.84(\mathrm{~s}, 6 \mathrm{H}), 3.76$ (s, 6H), $3.71(\mathrm{~s}, 3 \mathrm{H}) ;{ }^{13} \mathrm{C}-\mathrm{NMR}$ (DMSO-d $\left.d_{6}\right), \delta: 164.1,153.1,152.1$, $146.5,139.7,129.5,128.0,126.3,116.3,106.3,105.0,60.1,56.1,55.9 ;$ HR-MS: $\mathrm{C}_{20} \mathrm{H}_{23} \mathrm{O}_{7}[\mathrm{M}+$ $\mathrm{H}]^{+}$calculated $375.1438 \mathrm{~m} / \mathrm{z}$, found $375.1433 \mathrm{~m} / \mathrm{z}$.

5-Methyl-2-(propan-2-yl)phenyl (2E)-3-(3,4,5-trimethoxyphenyl)prop-2-enoate (9) [48]. Yield 74\%; Mp 103-105 ${ }^{\circ} \mathrm{C}$; IR (ATR, $\mathrm{cm}^{-1}$ ): 2973, 2936, 2838, 1724, 1638, 1580, 1502, 1461, 1432, $1414,1341,1308,1271,1241,1153,1119,1089,1060,934,980,922,872,828,820,785,755,740$, $667,654,609,586,575,545,527,517,495 ;{ }^{1} \mathrm{H}-\mathrm{NMR}\left(\mathrm{DMSO}-d_{6}\right), \delta: 7.80(\mathrm{~d}, J=15.8 \mathrm{~Hz}, 1 \mathrm{H})$, $7.27(\mathrm{~d}, J=8.2 \mathrm{~Hz}, 1 \mathrm{H}), 7.19(\mathrm{~s}, 2 \mathrm{H}), 7.07(\mathrm{dd}, J=8.2 \mathrm{~Hz}, J=1.4 \mathrm{~Hz}, 1 \mathrm{H}), 6.95(\mathrm{~d}, J=15.8 \mathrm{~Hz}$, $1 \mathrm{H}), 6.90(\mathrm{~d}, J=1.4 \mathrm{~Hz}, 1 \mathrm{H}), 3.84(\mathrm{~s}, 6 \mathrm{H}), 3.71(\mathrm{~s}, 3 \mathrm{H}), 2.96(\mathrm{spt}, J=6.9 \mathrm{~Hz}, 1 \mathrm{H}), 2.28(\mathrm{~s}, 3 \mathrm{H})$, $1.14(\mathrm{~d}, J=1.14 \mathrm{~Hz}, 6 \mathrm{H}) ;{ }^{13} \mathrm{C}-\mathrm{NMR}\left(\mathrm{DMSO}-d_{6}\right), \delta: 165.4,153.1,147.7,146.7,139.8,136.8$, $136.2,129.4,126.9,126.4,122.8,116.3,106.3,60.1,56.1,26.6,22.9,20.3 ;$ HR-MS: $\mathrm{C}_{22} \mathrm{H}_{27} \mathrm{O}_{5}$ $[\mathrm{M}+\mathrm{H}]^{+}$calculated $371.1853 \mathrm{~m} / \mathrm{z}$, found $371.1850 \mathrm{~m} / \mathrm{z}$.

4-(2,2,4,4-Butyl)phenyl (2E)-3-(3,4,5-trimethoxyphenyl)prop-2-enoate (10). Yield 70\%; Mp 150$152{ }^{\circ} \mathrm{C}$; IR (ATR, $\mathrm{cm}^{-1}$ ): 2983, 2957, 2905, 2839, 2824, 1716, 1632, 1584, 1504, 1455, 14419, 1395, 1365, 1341, 1326, 1272, 1240, 1200, 1166, 1157, 1123, 1093, 1018, 1009, 990, 948, 931, 918, $879,863,840,824,808,788,760,722,684,650,638,624,605,580,534,524,437 ;{ }^{1} \mathrm{H}-\mathrm{NMR}$ $\left(\mathrm{DMSO}-d_{6}\right), \delta: 7.79(\mathrm{~d}, J=15.8 \mathrm{~Hz}, 1 \mathrm{H}), 7.45-7.43(\mathrm{~m}, 2 \mathrm{H}), 7.17(\mathrm{~s}, 2 \mathrm{H}), 7.11-7.09(\mathrm{~m}, 2 \mathrm{H})$, $6.89(\mathrm{~d}, J=15.8 \mathrm{~Hz}, 1 \mathrm{H}), 3.83(\mathrm{~s}, 6 \mathrm{H}), 3.71(\mathrm{~s}, 3 \mathrm{H}), 1.73(\mathrm{~s}, 2 \mathrm{H}), 1.34(\mathrm{~s}, 6 \mathrm{H}), 0.70(\mathrm{~s}, 9 \mathrm{H})$; ${ }^{13}$ C-NMR (DMSO- $\left.d_{6}\right), \delta: 165.1,153.1,148.1,147.0,146.5,139.8,129.5,127.0,120.8,116.6$, 106.3, 60.1, 56.3, 56.1, 38.1, 32.1, 31.6, 31.4; HR-MS: $\mathrm{C}_{26} \mathrm{H}_{35} \mathrm{O}_{5}[\mathrm{M}+\mathrm{H}]^{+}$calculated 427.2479 $\mathrm{m} / \mathrm{z}$, found $427.2469 \mathrm{~m} / \mathrm{z}$.

Benzyl (2E)-3-(3,4,5-trimethoxyphenyl)prop-2-enoate (11) [14]. Yield 48\%; Mp 92-95 ${ }^{\circ} \mathrm{C}$; IR (ATR, $\mathrm{cm}^{-1}$ ): 2994, 2960, 2941, 2841, 1701, 1636, 1582, 1503, 1472, 1455, 1430, 1418, 1344, 1322, 1308, 1273, 1246, 1194, 1185, 1166, 1149, 1123, 1043, 1001, 976, 917, 844, 827, 787, 764,

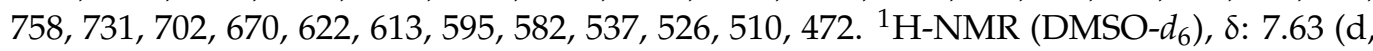
$J=15.8 \mathrm{~Hz}, 1 \mathrm{H}), 7.44-7.39(\mathrm{~m}, 4 \mathrm{H}), 7.37-7.34(\mathrm{~m}, 1 \mathrm{H}), 7.09(\mathrm{~s}, 2 \mathrm{H}), 6.73(\mathrm{~d}, J=16.5 \mathrm{~Hz}, 1 \mathrm{H})$, $5.22(\mathrm{~s}, 2 \mathrm{H}), 3.81(\mathrm{~s}, 6 \mathrm{H}), 3.68(\mathrm{~s}, 3 \mathrm{H}) ;{ }^{13} \mathrm{C}-\mathrm{NMR}$ (DMSO- $\left.d_{6}\right), \delta: 166.2,153.1,145.1,139.5$, $136.2,129.6,128.5,128.2,128.1,117.1,106.0,65.6,60.1,56.0$; HR-MS: $\mathrm{C}_{19} \mathrm{H}_{21} \mathrm{O}_{5}[\mathrm{M}+\mathrm{H}]^{+}$ calculated $329.1384 \mathrm{~m} / \mathrm{z}$, found $329.1383 \mathrm{~m} / \mathrm{z}$. 
2-Propoxyethyl (2E)-3-(3,4,5-trimethoxyphenyl)prop-2-enoate (12). Yield 48\%; Mp 67-69 ${ }^{\circ} \mathrm{C}$; IR (ATR, $\left.\mathrm{cm}^{-1}\right)$ : 3003, 2952, 2870, 2843, 1701, 1639, 1583, 1506, 1464, 1450, 1417, 1389, 1346, 1318, 1306, $1272,1246,1176,1152,1124,1100,1064,1049,1024,996,980,901,887,847,817,791,766,671,621$, 611, 593, 567, 527, 497; ${ }^{1} \mathrm{H}-\mathrm{NMR}\left(\mathrm{DMSO}-d_{6}\right), \delta: 7.60$ (d, J = 15.8 Hz, 1H), 7.09 (s, 2H), 6.70 (d, $J=15.8 \mathrm{~Hz}, 1 \mathrm{H}), 4.26(\mathrm{~m}, 2 \mathrm{H}), 3.82(\mathrm{~s}, 6 \mathrm{H}), 3.69(\mathrm{~s}, 3 \mathrm{H}), 3.63-3.61(\mathrm{~m}, 2 \mathrm{H}), 3.38(\mathrm{t}, J=6.5 \mathrm{~Hz}, 2 \mathrm{H})$, $1.51(\mathrm{sxt}, J=6.9 \mathrm{~Hz}, 2 \mathrm{H}), 0.86(\mathrm{t}, J=7.6 \mathrm{~Hz}, 3 \mathrm{H}) ;{ }^{13} \mathrm{C}-\mathrm{NMR}\left(\mathrm{DMSO}-d_{6}\right) \delta: 166.8,153.3,145.2,139.7$, $129.9,117.5,106.1,72.1,68.2,63.6,60.4,56.3,22.6,10.7$; HR-MS: $\mathrm{C}_{17} \mathrm{H}_{25} \mathrm{O}_{6}[\mathrm{M}+\mathrm{H}]^{+}$calculated $325.1646 \mathrm{~m} / \mathrm{z}$, found $325.1639 \mathrm{~m} / \mathrm{z}$.

\subsection{Evaluating In Vitro AChE and BChE-Inhibition Potencies}

The ability of all the prepared compounds to inhibit AChE from electric eel (Electrophorus electricus) and BChE from equine serum (both purchased from Sigma, St. Louis, MO, USA) was determined in vitro using a modified Ellman's method, as described previously [49-53].

\subsection{Measurement of Inhibition Type}

Compound 7 was used for the determination of the type of inhibition. The measuring procedure was analogue to the determination of $\mathrm{IC}_{50}$. For the measurement, the following concentrations of the substrate were used: 20, 40,60 and $80 \mu \mathrm{M}$. Methodology was performed as the method described previously [54,55].

\subsection{In Vitro Viability Assay}

The safety of the investigated compounds (effect on cell viability) was evaluated on two different cell lines: the adherent human synovial cell line SW982 (ATCC, Manassas, VA, USA) and suspension human genetically modified leukemia cell line THP1-Blue ${ }^{\mathrm{TM}} \mathrm{NF}-\kappa \mathrm{B}$ (Invivogen, San Diego, CA, USA). Screening was performed as described previously [44,45].

\section{Results and Discussion}

\subsection{Chemistry and Physicochemical Properties}

All the investigated compounds 1-12 were synthesized according to Scheme 1 . The carboxyl group of the starting TMCA was converted with thionyl chloride to the acyl chloride, which, upon subsequent reaction with the appropriate alcohol/phenol, yielded the targeted ester. Both reactions (conversion to the acid chloride and subsequent ester formation) were performed in dry toluene under reflux. All the compounds were recrystallized from ethanol.

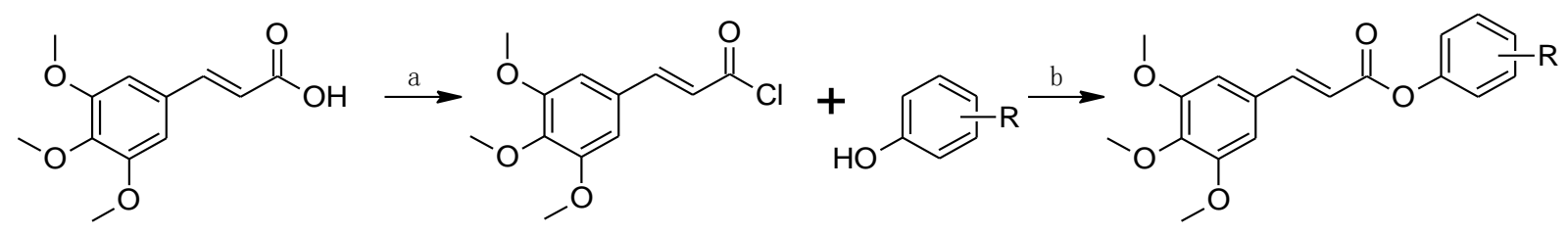

Scheme 1. Synthesis of aryl (2E)-3-(3,4,5-trimethoxyphenyl)prop-2-enoates 1-12. Reagents and conditions: a: $\mathrm{SOCl}_{2}$, dry toluene, reflux $1 \mathrm{~h} ; \mathrm{b}: \mathrm{Et}_{3} \mathrm{~N}$, dry toluene, reflux $1 \mathrm{~h}$.

Lipophilicity (expressed as $\log P$ values) of compounds 1-12, electronic $\sigma$ parameters of individual ester substituents (-R) and molar volumes (MV $\left(\mathrm{cm}^{3}\right)$ ) of individual ester substituents $(-\mathrm{R})$ were used as physicochemical descriptors characterizing individual investigated compounds. The program ACD/Percepta ver. 2012 was used for all predicted values (see Table 1). As expected, TMCA showed the lowest $\log P$. Of the target esters, aliphatic derivative 12 ( $\mathrm{R}=2$-propoxyethyl (PPXE), $\log P=2.48)$ and compound $8\left(\mathrm{R}=2,6-\mathrm{OCH}_{3}-\mathrm{Ph}, \log P=2.90\right)$ were the least lipophilic compounds. Compound $10(\mathrm{R}=4-(2,4,4$-trimethylpentan-2-yl)phenyl (TMPP), $\log P=6.43$ ) showed the highest predicted lipophilicity. The fact that methoxyphenyl or benzyl substituents have lower lipophilicity than their "parent" phenyl substituent was described, for example, in [56-61]. The lowest electronic $\sigma$ parameters for individual ester 
substituents were predicted for compound $8(\sigma=0.09)$; the highest electron-withdrawing effect was observed for compounds substituted in the ortho position of the phenyl ring by fluorine (6, $\sigma=1.02)$ and chlorine $(7, \sigma=1.05)$. Thus, it can be stated that most ester substituents possess electron-withdrawing properties in the range from approx. 0.2 to 1.05 . The bulkiness of the individual ester substituents, expressed as molar volume, was logically the smallest for the unsubstituted phenyl ester $\mathbf{1}\left(\mathrm{MV}=80.88 \mathrm{~cm}^{3}\right)$; on the contrary, compound $10(\mathrm{R}=\mathrm{TMPP})$ has by far the largest volume $\mathrm{MV}=213.31 \mathrm{~cm}^{3}$ within the whole series. In addition, compound 9 ( $\mathrm{R}=2$-isopropyl-5-methylphenyl (IPMP)) has the second largest volume within the investigated series of esters, with an MV value of $146.96 \mathrm{~cm}^{3}$.

Table 1. Structures of 3,4,5-trimethoxycinnamic acid (TMCA) and its discussed ring-substituted esters 1-12; calculated values of $\log P$, electronic $\sigma$ parameters of $\mathrm{R}$ substituents and molar volume $\left(\mathrm{MV}\left(\mathrm{cm}^{3}\right)\right)$ of R substituents; in vitro AChE and $\mathrm{BChE}$ inhibition $\left(\mathrm{IC}_{50}(\mu \mathrm{M})\right)$ compared with standards rivastigmine (RIV) and galantamine (GLT) and in vitro viability (SW982, THP1-Blue ${ }^{\mathrm{TM}} \mathrm{NF}-\mathrm{kB}$ ) assays ( $\mathrm{IC}_{50}(\mu \mathrm{M})$ after $24 \mathrm{~h}$ and $72 \mathrm{~h}$ incubation) of investigated compounds. Cholinesterase inhibition is expressed as the mean $\pm \mathrm{SD}$ ( $n=3$ experiments).<smiles>[R]OC(=O)/C=C/c1cc(OC)c(OC)c(OC)c1</smiles>

\begin{tabular}{|c|c|c|c|c|c|c|c|c|c|c|}
\hline \multirow{3}{*}{$\begin{array}{c}\text { Comp. } \\
1\end{array}$} & \multirow{3}{*}{$\begin{array}{l}\mathbf{R} \\
\\
\text { II }\end{array}$} & \multirow{3}{*}{$\begin{array}{c}\log P^{a} \\
3.23\end{array}$} & \multirow{3}{*}{$\begin{array}{c}\sigma_{(\mathbf{R})}{ }^{a} \\
0.60\end{array}$} & \multirow{3}{*}{$\begin{array}{c}\mathbf{M V}_{(\mathrm{R})}{ }^{a} \\
80.88\end{array}$} & \multirow{3}{*}{$\begin{array}{c}\text { AChE } \\
55.01 \pm 1.05\end{array}$} & \multirow{3}{*}{$\begin{array}{c}\text { BChE } \\
74.74 \pm 1.36\end{array}$} & \multirow{3}{*}{$\begin{array}{c}\mathrm{SI}^{b} \\
0.74\end{array}$} & \multicolumn{3}{|c|}{ Tox $\mathrm{IC}_{50}(\mu \mathrm{M})$} \\
\hline & & & & & & & & \multirow{2}{*}{$\begin{array}{l}\begin{array}{l}\text { SW982 } \\
(72 \mathrm{~h})\end{array} \\
>30\end{array}$} & \multicolumn{2}{|c|}{$\begin{array}{c}\text { THP } \\
(72 \text { h) }(24 \text { h) }\end{array}$} \\
\hline & & & & & & & & & $>30$ & $>30$ \\
\hline 2 & & 3.19 & 0.36 & 103.61 & $57.50 \pm 0.02$ & $117.91 \pm 2.81$ & 0.49 & $>30$ & $>30$ & $>30$ \\
\hline 3 & & 3.49 & 0.46 & 97.11 & $69.57 \pm 0.65$ & $81.12 \pm 1.92$ & 0.86 & $>30$ & $>30$ & $>30$ \\
\hline 4 & & 3.34 & 0.62 & 85.90 & $65.22 \pm 3.70$ & $72.58 \pm 0.27$ & 0.90 & $>30$ & $>30$ & $>30$ \\
\hline 5 & & 3.79 & 0.74 & 94.14 & $46.10 \pm 1.07$ & $99.25 \pm 1.72$ & 0.46 & $>30$ & $>30$ & $\sim 54$ \\
\hline 6 & & 3.15 & 1.02 & 85.90 & $75.79 \pm 0.37$ & $44.41 \pm 1.95$ & 1.71 & $>30$ & $>30$ & $>60$ \\
\hline 7 & & 3.72 & 1.05 & 92.25 & $46.18 \pm 0.81$ & $32.46 \pm 0.76$ & 1.42 & $>30$ & $>30$ & $>60$ \\
\hline 8 & & 2.90 & 0.09 & 126.10 & $50.45 \pm 0.61$ & $166.20 \pm 8.15$ & 0.30 & $>30$ & $>30$ & $>30$ \\
\hline 9 & & 4.79 & 0.60 & 146.96 & $46.59 \pm 0.60$ & $130.92 \pm 5.08$ & 0.36 & $>30$ & $>30$ & $>60$ \\
\hline 10 & & 6.43 & 0.45 & 213.31 & $53.41 \pm 1.86$ & $376.63 \pm 32.73$ & 0.14 & $>30$ & $>30$ & $>30$ \\
\hline
\end{tabular}


Table 1. Cont.

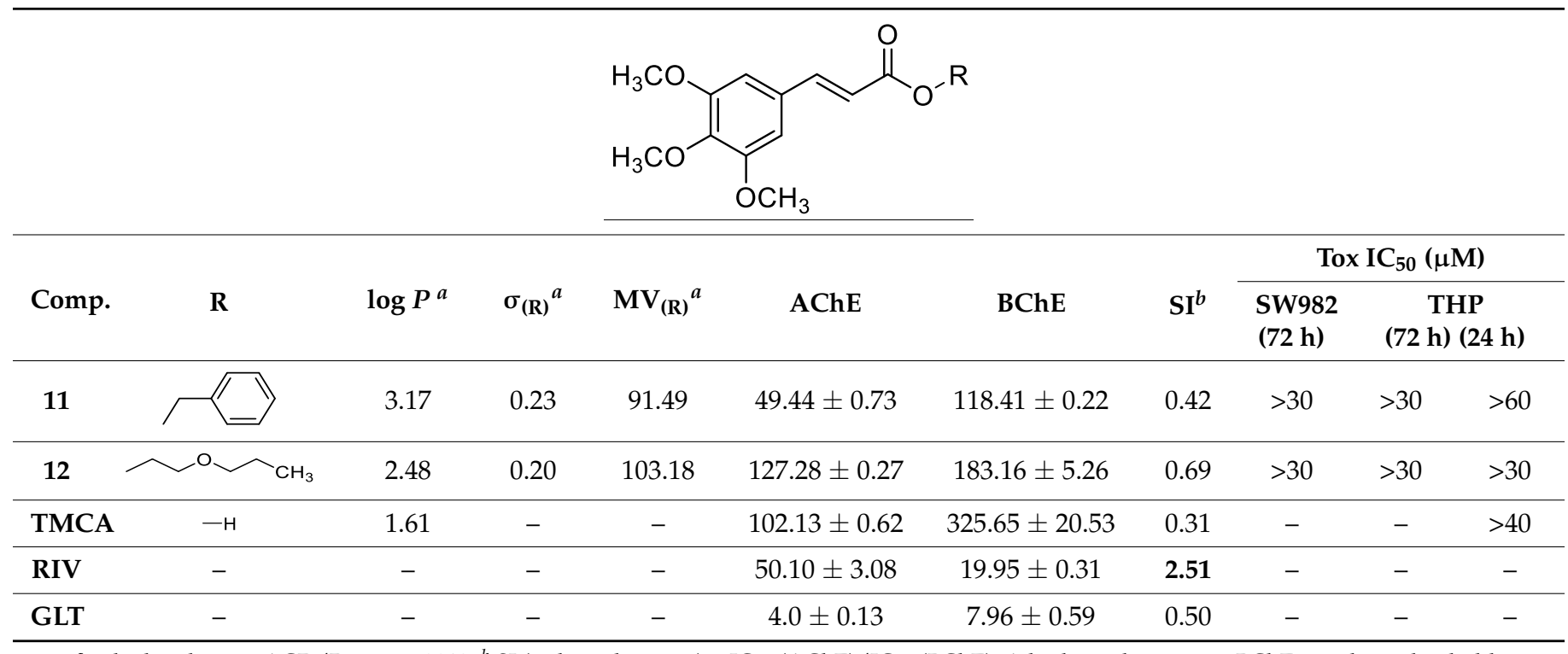

${ }^{a}$ calculated using $\mathrm{ACD} /$ Percepta 2012; ${ }^{b} \mathrm{SI}$ (index selectivity) $=\mathrm{IC}_{50}(\mathrm{AChE}) / \mathrm{IC}_{50}(\mathrm{BChE})$. A higher selectivity to $\mathrm{BChE}$ is indicated in bold.

\subsection{In Vitro Evaluation of AChE- and BChE-Inhibiting Activity}

All the esters were evaluated for their ability to inhibit AChE and BChE. This activity was compared with parent TMCA and the clinically used drugs rivastigmine (RIV) and galantamine (GLT). RIV (Figure 1) is a carbamate derivative structurally derived from physostigmine. The exact mechanism of RIV has not been fully established, but RIV is thought to be a classical acylation pseudo-reversible carbamate cholinesterase inhibitor that inhibits both $\mathrm{AChE}$ and $\mathrm{BChE}$, thereby preventing hydrolysis of acetylcholine, leading to increased acetylcholine concentrations at cholinergic synapses. The anticholinesterase activity of rivastigmine is relatively specific for brain $\mathrm{AChE}$ and $\mathrm{BChE}$ compared to activities in peripheral tissues [62]. GAL (Figure 1) is a tertiary alkaloid extracted from Galanthus nivalis (Amaryllidaceae). It is a centrally and peripherally acting non-acylated competitive reversible inhibitor of muscle and brain $\mathrm{AChE}$, thereby increasing cholinergic tone. In addition, it also acts as a positive allosteric modulator of neuronal nicotinic acetylcholine receptors, which may also contribute to ameliorating $\mathrm{AD}$ symptoms [63]. This activity was expressed as $\mathrm{IC}_{50}(\mu \mathrm{M})$ (concentration of inhibitor that was required for $50 \%$ inhibition of the enzymes).

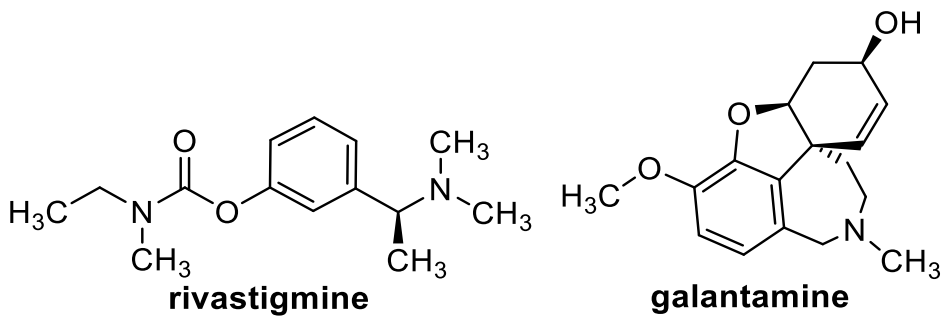

Figure 1. Structures of approved drugs used as standards in this work.

Based on the results summarized in Table 1, it can be stated that cholinesterase's inhibitory activity is moderate, but several of the most active compounds showed comparable inhibition of AChE with RIV. Compounds $5(\mathrm{R}=4-\mathrm{Br}-\mathrm{Ph}), 7(\mathrm{R}=2-\mathrm{Cl}-\mathrm{Ph})$ and 9 $(\mathrm{R}=\mathrm{IPMP})$ showed $\mathrm{IC}_{50} \mathrm{ca} .46 \mu \mathrm{M}$ and compound $11(\mathrm{R}=\mathrm{Bn})$ had $\mathrm{IC}_{50}=49.44 \mu \mathrm{M}$. On the other hand, only two compounds demonstrated more significant $\mathrm{BChE}$ inhibition: ester $7\left(\mathrm{R}=2-\mathrm{Cl}-\mathrm{Ph}, \mathrm{IC}_{50}=32.46 \mu \mathrm{M}\right)$ and $6\left(\mathrm{R}=2-\mathrm{F}-\mathrm{Ph}, \mathrm{IC}_{50}=44.41 \mu \mathrm{M}\right)$. Moreover, it should be noted that the selectivity indices (increased $\mathrm{BChE}$ inhibition in comparison with $\mathrm{AChE}$ 
inhibition) for these two compounds are 1.42 and 1.71, respectively, which makes these two compounds valuable, as mentioned above.

The dependences of the $\mathrm{AChE}$ inhibition of the compounds expressed as $\log \left(1 / \mathrm{IC}_{50}(\mathrm{M})\right)$ on lipophilicity; expressed as $\log P$, electronic $\sigma$ parameters and bulkiness of the individual ester substituents; and expressed as MV are illustrated in Figure 2A-C. While it is evident that the effect of $\sigma$ parameters on AChE inhibition is negligible (see Figure 2B), it can be seen that lipophilicity in the $\log P 4-5$ range is advantageous for higher activity (Figure 2A), similar to the bulkiness of ester substituents in the MV range from 94 to $146 \mathrm{~cm}^{3}$ (Figure 2C).

The dependences of the $\mathrm{BChE}$ inhibition of the investigated compounds expressed as $\log \left(1 / \mathrm{IC}_{50}(\mathrm{M})\right)$ on lipophilicity expressed as $\log P$, electronic $\sigma$ parameters and bulkiness of the individual ester substituents are illustrated in Figure $3 \mathrm{~A}-\mathrm{C}$. In contrast to $\mathrm{AChE}$ inhibition, a bilinear dependence of $\mathrm{BChE}$ inhibition on $\log P$ values can be observed in Figure 3A, with a maximum ca. $\log P=3.7$. From the dependence shown in Figure 3B, a linear dependence is clearly seen, where the activity increases with the electron-withdrawing properties of the ester substituents. The bilinear trend (increasing BChE inhibition to $\mathrm{MV}=92 \mathrm{~cm}^{3}(7, \mathrm{R}=2-\mathrm{Cl}-\mathrm{Ph})$ followed by decreasing activity with increasing ester bulkiness) can also be traced to Figure 3C.
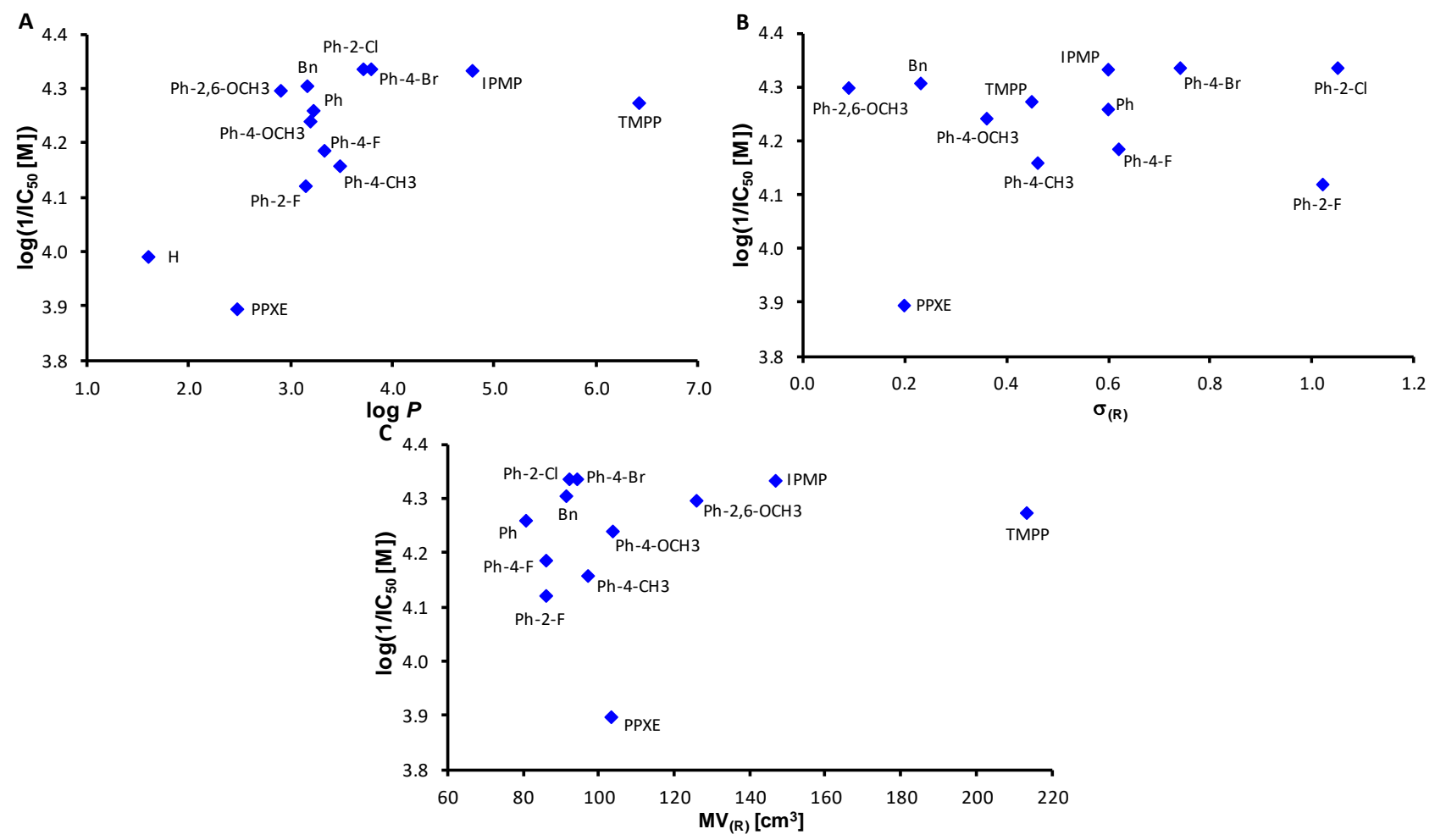

Figure 2. Dependences of AChE-inhibiting activity $\log \left(1 / \mathrm{IC}_{50}(\mathrm{M})\right)$ of all compounds 1-13 on lipophilicity expressed as log $P(\mathbf{A})$, electronic $\sigma$ parameters and bulkiness expressed as molar volume (MV) of whole $O$-part of individual esters (B,C). IPMP = 2-isopropyl-5-methylphenyl, TMPP = 4-(2,4,4-trimethylpentan-2-yl)phenyl, PPXE = 2-propoxyethyl.

It is important to specify that while for AChE inhibition, the position of the substituent on the phenyl ring does not appear to be absolutely crucial (para substituted isomers have only slightly better activity; compare para vs. ortho position of the substituents of compounds $\mathbf{5}$ and $\mathbf{7}$ or $\mathbf{4}$ and 6), for $\mathrm{BChE}$ inhibition, it is absolutely the fundamental position of the substituent in the ortho position. The same observations were found recently, e.g., in $[53,56,64-68]$. 

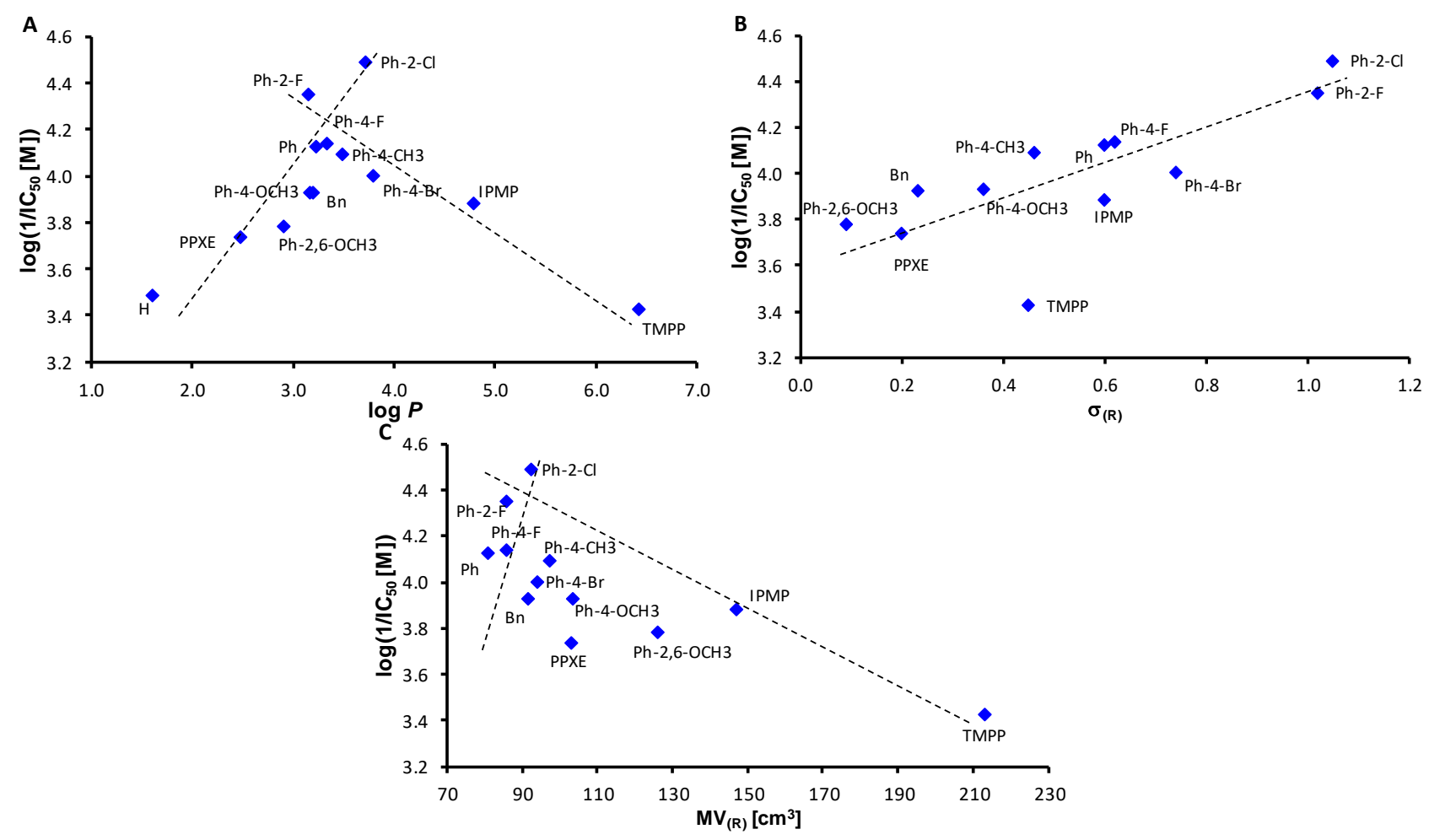

Figure 3. Dependences of BChE-inhibiting activity $\log \left(1 / \mathrm{IC}_{50}(\mathrm{M})\right)$ of all compounds 1-13 on lipophilicity expressed as $\log$ $P(\mathbf{A})$, electronic $\sigma$ parameters and bulkiness expressed as molar volume (MV) of whole $O$-part of individual esters (B,C). IPMP = 2-isopropyl-5-methylphenyl, TMPP = 4-(2,4,4-trimethylpentan-2-yl)phenyl, PPXE = 2-propoxyethyl.

\section{Type of Inhibition}

The reversible inhibitors are usually divided into four groups: competitive, noncompetitive, uncompetitive and mixed. The mechanism of action of the inhibitor can be determined using the Lineweaver-Burk (L-B) plot and comparing the kinetic parametersMichaelis constant $\left(K_{\mathrm{M}}\right)$ and maximum velocity $\left(V_{\mathrm{m}}\right)$ of the uninhibited and inhibited reaction. The competitive inhibitor increases $K_{\mathrm{M}}$ by interfering with the active site of the enzyme but does not affect $V_{\mathrm{m}}$. All lines in the L-B plot intersect at the same point on the $y$-axis. The non-competitive inhibitor does not change $K_{\mathrm{M}}$ but decreases $V_{\mathrm{m}}$. All lines in the $\mathrm{L}-\mathrm{B}$ plot intersect at the same point on the $x$-axis. The uncompetitive inhibitor decreases $K_{\mathrm{M}}$ and $V_{\mathrm{m}}$, but their ratio is the same as for the uninhibited reaction. All lines in the L-B plot are parallel. The inhibitor acting via the mixed type of inhibition changes $K_{\mathrm{M}}, V_{\mathrm{m}}$ and their ratio compared to the reaction in the absence of the inhibitor. All lines in the L-B plot intersect in quadrant II or III [54].

L-B plots for the most effective compound 7. inhibiting AChE and BChE, are depicted on Figures $4 \mathrm{~A}$ and $5 \mathrm{~A}$. Based on the L-B plots, ester 7 acts via the mixed type of inhibition. Moreover, the inhibition constant $K_{\mathrm{i}}$ (i.e., dissociation constant of complex enzyme-inhibitor) was calculated using the regression equation of the dependence of the slope (L-B plot) vs. the concentration of the inhibitor (Figures 4B and 5B).

\subsection{In Vitro Cell Viability}

To exclude the potential cytotoxic effect of the tested compounds, their influence on cell viability was evaluated on two different cell lines_adherent human synovial cell line SW982 and suspension human genetically modified leukemia cell line THP1-Blue ${ }^{\mathrm{TM}} \mathrm{NF}-$ $\kappa \mathrm{B}$. All of the used compounds slightly reduced cell viability (measured as mitochondrial metabolism) at the highest concentration of $30 \mu \mathrm{M}$ after 24 and $72 \mathrm{~h}$. However, the observed relative cell viability was $>70 \%$ for both cell lines and all compounds. Moreover, the most 
active $\mathrm{AChE}$ and $\mathrm{BChE}$ inhibitors 6, 7, 9, and 11 did not decrease the relative viability of THP1-Blue ${ }^{\mathrm{TM}}$ NF- $\mathrm{\kappa B}$ cells under $50 \%$ after $24 \mathrm{~h}$ incubation, when they were used at the concentration of $60 \mu \mathrm{M}$. The compound 5 with the most "cytotoxic" behavior had an $\mathrm{IC}_{50}$ value for THP1-Blue ${ }^{\mathrm{TM}} \mathrm{NF}-\mathrm{kB}$ of $\sim 54 \mu \mathrm{M}$ after $24 \mathrm{~h}$ incubation.

A

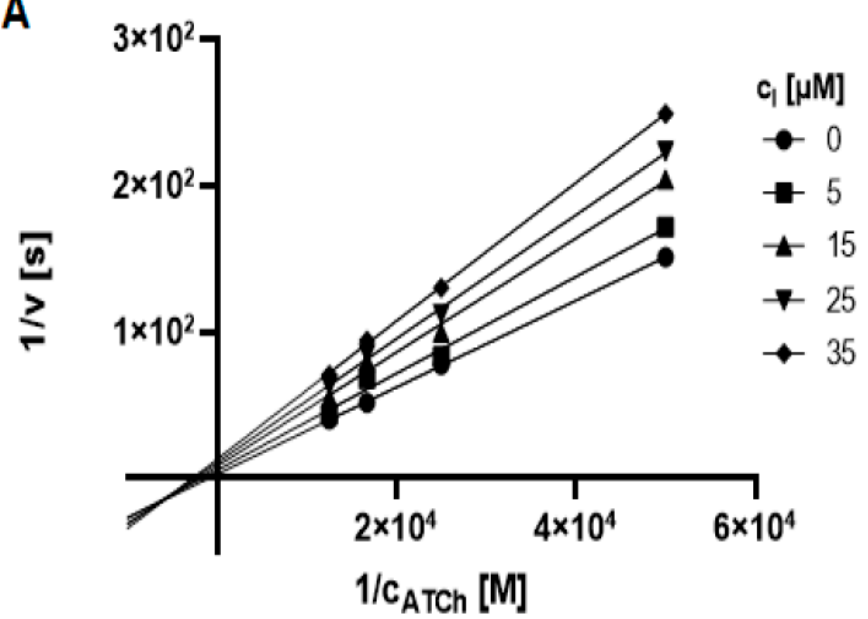

B

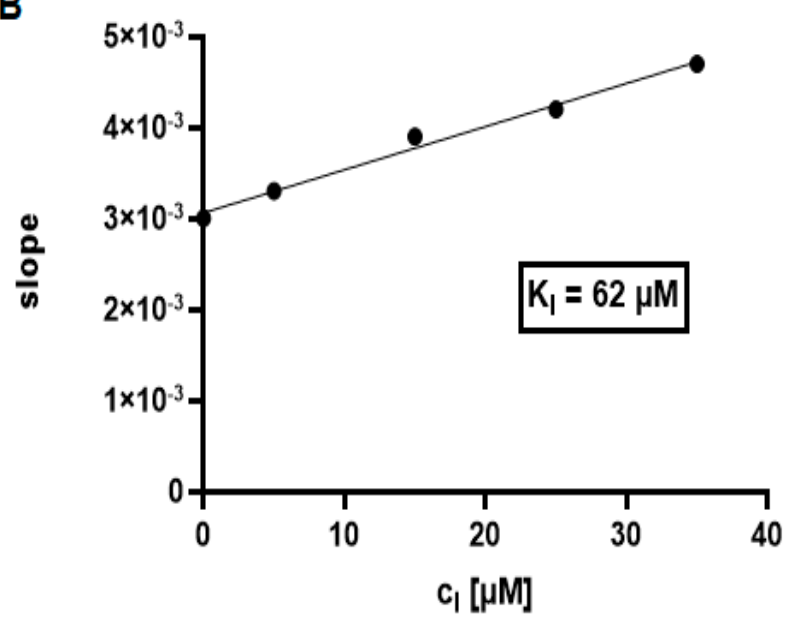

Figure 4. Lineweaver-Burk plot for compound 7 inhibiting AChE. The measurements were performed in duplicate and the points are expressed as average values (A). Dependence of the slope (Lineweaver-Burk plot) vs. concentration of inhibitor (combination AChE-7) (B).
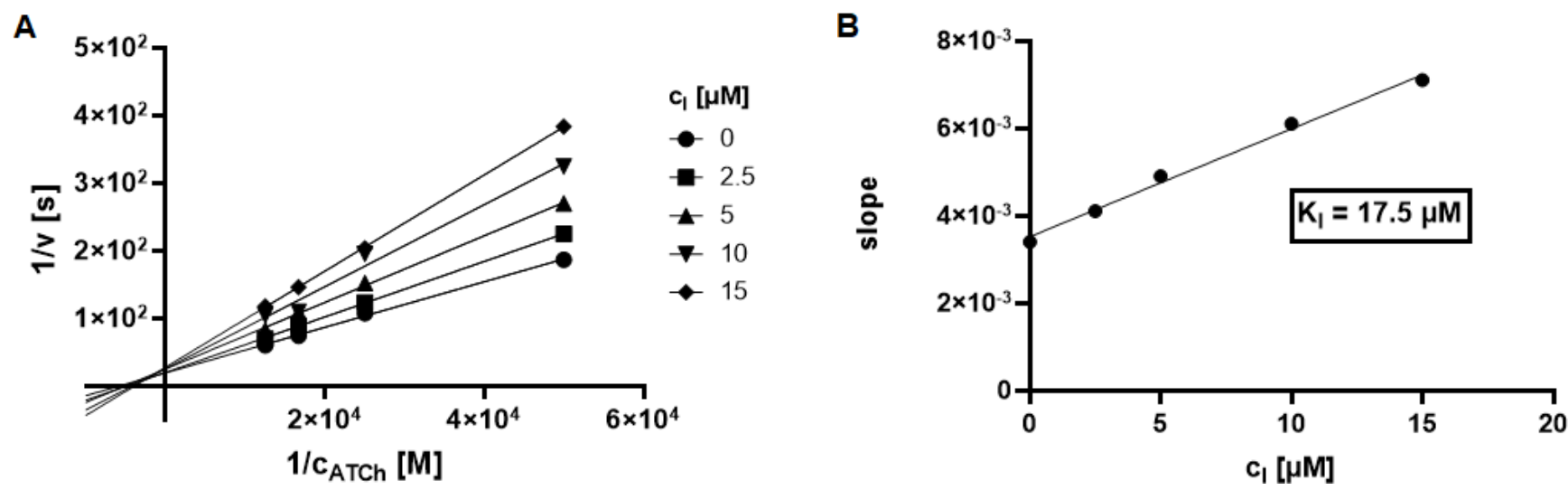

Figure 5. Lineweaver-Burk plot for compound 7 inhibiting BChE. The measurements were performed in duplicate and points are expressed as average values (A). Dependence of slope (Lineweaver-Burk plot) vs. concentration of inhibitor (combination BChE-7) (B).

\section{Conclusions}

A series of twelve trimethoxycinnamic acid esters was prepared and characterized. The starting trimethoxycinnamic acid and all prepared esters were tested for their ability to inhibit in vitro AChE and BChE. 2-Chlorophenyl (2E)-3-(3,4,5-trimethoxyphenyl)- prop2-enoate (7) showed the highest activity against both $\mathrm{AChE}\left(\mathrm{IC}_{50}=46.18 \mu \mathrm{M}\right)$ and $\mathrm{BChE}$ $\left(\mathrm{IC}_{50}=32.46 \mu \mathrm{M}\right)$. However, $(2 E)$-3-(3,4,5-trimethoxyphenyl)prop-2-enoate (6) had the best BChE selectivity index (SI = 1.71). Based on the Lineweaver-Burk plot for compound 7, it was determined that it acts via a mixed type of inhibition for both enzymes. The position of the substituent on the phenyl ring does not appear to be essential for AChE inhibition, nor have significant effects for the lipophilicity, bulkiness or electronic parameters of the substituents on AChE activity been found. On the other hand, for BChE-inhibiting activity, the absolutely essential position of the substituent is in the ortho position. Additionally, 
for BChE inhibition, an increase in activity can be observed with an increasing electronwithdrawing effect of the substituent, which, at the same time, has lipophilicity expressed as $\log P$ ca. 3.7 and rather less bulkiness (ca. $\mathrm{MV}=92 \mathrm{~cm}^{3}$ ). All studied compounds were evaluated for their in vitro cytotoxicity on two human cell lines and the active compounds were found to be insignificantly cytotoxic, underlining the importance of studying these small molecules in the future.

Author Contributions: J.K. and T.S. synthesized and characterized the compounds. M.O. performed analytical measurement. S.S., K.S., A.I. and J.H. performed biological screening. J.K. and J.J. designed the compounds. J.K., S.S., J.H. and J.J. wrote the paper. All authors have read and agreed to the published version of the manuscript.

Funding: This study was supported by the Slovak Research and Development Agency (project APVV-18-0302). T.S. was supported by Palacky University in Olomouc (IGA_PrF_2021_032). The HPLC/HRMS system forms a part of the National Infrastructure CzeCOS ProCES CZ.02.1.01/0.0/0.0/ 16_013/0001609; M.O. was supported by SustES (CZ.02.1.01/0.0/0.0/16_019/0000797). The work of J.H. was also supported by the Ministry of Education, Youth and Sports of the Czech Republic under the project "FIT" CZ.02.1.01/0.0/0.0/15_003/0000495, and Czech Ministry of Agriculture grant no. RO0518. The authors wish to acknowledge financial support from the University of Pardubice, Faculty of Chemical Technology.

Institutional Review Board Statement: Not applicable.

Informed Consent Statement: Not applicable.

Data Availability Statement: Data are contained within the article.

Conflicts of Interest: The authors declare no conflict of interest.

\section{References}

1. Newman, D.J.; Cragg, G.M. Natural products as sources of new drugs from 1981 to 2014. J. Nat. Prod. 2016, 79, 629-661. [CrossRef]

2. Kralova, K.; Jampilek, J. Responses of Medicinal and aromatic plants to engineered nanoparticles. Appl. Sci. 2021, 11, 1813. [CrossRef]

3. Jampilek, J. Recent advances in design of potential quinoxaline anti-infectives. Curr. Med. Chem. 2014, 21, 4347-4373. [CrossRef] [PubMed]

4. Jampilek, J. Design of antimalarial agents based on natural products. Curr. Org. Chem. 2017, 21, 1824-1846. [CrossRef]

5. Dolab, J.G.; Lima, B.; Spaczynska, E.; Kos, J.; Cano, N.H.; Feresin, G.; Tapia, A.; Garibotto, F.; Petenatti, E.; Olivella, M.; et al. Antimicrobial activity of Annona emarginata (Schltdl.) H. Rainer and most active isolated compound against clinically important bacteria. Molecules 2018, 23, 1187. [CrossRef]

6. Jampilek, J. Design and discovery of new antibacterial agents: Advances, perspectives, challenges. Curr. Med. Chem. 2018, 25, 4972-5006. [CrossRef] [PubMed]

7. Shuab, R.; Lone, R.; Koul, K.K. Cinnamate and cinnamate derivatives in plants. Acta Physiol. Plant 2016, 38, 64. [CrossRef]

8. Ruwizhi, N.; Aderibigbe, B.A. Cinnamic acid derivatives and their biological efficacy. Int. J. Mol. Sci. 2020, 21, 5712. [CrossRef]

9. 3,4,5-Trimethoxycinnamic Acid. EMBL-EBI, Hinxton, UK. Available online: https://www.ebi.ac.uk/chebi/searchId.do?chebiId= CHEBI:566519 (accessed on 19 March 2021).

10. Klein, L.C.; de Andrade, S.F.; Filho, V.C. A pharmacognostic approach to the Polygala genus: Phytochemical and pharmacological aspects. Chem. Biodivers. 2012, 9, 181-209. [CrossRef]

11. May, B.H.; Lu, C.; Lu, Y.; Zhang, A.L.; Xue, C.C. Chinese herbs for memory disorders: A review and systematic analysis of classical herbal literature. J. Acupunct. Meridian Stud. 2013, 6, 2-11. [CrossRef]

12. Jung, J.C.; Min, D.; Kim, H.; Jang, S.; Lee, Y.; Park, W.K.; Khan, I.A.; Moon, H.I.; Jung, M.; Oh, S. Design, synthesis, and biological evaluation of 3,4,5-trimethoxyphenyl acrylamides as antinarcotic agents. J. Enzyme Inhib. Med. Chem. 2010, 25, 38-43. [CrossRef]

13. Chen, C.Y.; Wei, X.D.; Chen, C.R. 3,4,5-Trimethoxycinnamic acid, one of the constituents of Polygalae radix exerts anti-seizure effects by modulating GABAAergic systems in mice. J. Pharmacol. Sci. 2016, 131, 1-5. [CrossRef]

14. Jung, J.C.; Moon, S.; Min, D.; Park, W.K.; Jung, M.; Oh, S. Synthesis and evaluation of a series of 3,4,5-trimethoxycinnamic acid derivatives as potential antinarcotic agents. Chem. Biol. Drug Des. 2013, 81, 389-398. [CrossRef]

15. Leslie, B.J.; Holaday, C.R.; Nguyen, T.; Hergenrother, P.J. Phenylcinnamides as novel antimitotic agents. J. Med. Chem. 2010, 53, 3964-3972. [CrossRef] [PubMed]

16. Kamal, A.; Bajee, S.; Nayak, V.L.; Rao, A.V.S.; Nagaraju, B.; Reddy, C.R.; Sopanrao, K.J.; Alarifi, A. Synthesis and biological evaluation of arylcinnamide linked combretastatin-A4 hybrids as tubulin polymerization inhibitors and apoptosis inducing agents. Bioorg. Med. Chem. Lett. 2016, 26, 2957-2964. [CrossRef] [PubMed] 
17. Wong, I.L.K.; Wang, B.C.; Yuan, J.; Duan, L.X.; Liu, Z.; Liu, T.; Li, X.M.; Hu, X.; Zhang, X.Y.; Jiang, T. Potent and nontoxic chemosensitizer of P-glycoprotein mediated multidrug resistance in cancer: Synthesis and evaluation of methylated epigallocatechin, gallocatechin, and dihydromyricetin derivatives. J. Med. Chem. 2015, 58, 529-4549. [CrossRef]

18. Xu, C.C.; Deng, T.; Fan, M.L.; Lv, W.B.; Liu, J.H.; Yu, B.Y. Synthesis and in vitro antitumor evaluation of dihydroartemisinincinnamic acid ester derivatives. Eur. J. Med. Chem. 2016, 107, 192-203. [CrossRef] [PubMed]

19. Wang, L.J.; Geng, C.A.; Ma, Y.B.; Luo, J.; Huang, X.Y.; Chen, H.; Zhou, N.J.; Zhang, X.M.; Chen, J.J. Design, synthesis, and molecular hybrids of caudatin and cinnamic acids as novel anti-hepatitis B virus agents. Eur. J. Med. Chem. 2012, 54, 352-365. [CrossRef] [PubMed]

20. Qurat-Ul-Ain, S.; Wang, W.; Yang, M.; Du, N.; Wan, S.; Zhang, L.; Jiang, T. Anomeric selectivity and influenza A virus inhibition study on methoxylated analogues of pentagalloylglucose. Carbohydr. Res. 2014, 402, 152-157. [CrossRef]

21. Zhao, Z.; Song, H.; Xie, J.; Liu, T.; Zhao, X.; Chen, X.; He, X.; Wu, S.; Zhang, Y.; Zheng, X. Research progress in the biological activities of 3,4,5-trimethoxycinnamic acid (TMCA) derivatives. Eur. J. Med. Chem. 2019, 173, 213-227. [CrossRef]

22. Kumar, S.; Singh, B.K.; Arya, P.; Malhotra, S.; Thimmulappa, R.; Prasad, A.K.; Van der Eycken, E.; Olsen, C.E.; Depass, A.L.; Biswal, S. Novel natural product-based cinnamates and their thio and thiono analogs as potent inhibitors of cell adhesion molecules on human endothelial cells. Eur. J. Med. Chem. 2011, 46, 5498-5511. [CrossRef] [PubMed]

23. Zhang, C.; Du, Q.Y.; Chen, L.D.; Wu, W.H.; Liao, S.Y.; Yu, L.H.; Liang, X.T. Design, synthesis and evaluation of novel tacrinemultialkoxybenzene hybrids as multi-targeted compounds against Alzheimer's disease. Eur. J. Med. Chem. 2016, 116, 200-209. [CrossRef]

24. Peng, S.; Zhang, B.; Meng, X.; Yao, J.; Fang, J. Synthesis of piperlongumine analogues and discovery of nuclear factor erythroid 2-related factor 2 (Nrf2) activators as potential neuroprotective agents. J. Med. Chem. 2015, 58, 5242-5255. [CrossRef]

25. Zhao, Z.; Bai, Y.; Xie, J.; Chen, X.; He, X.; Sun, Y.; Bai, Y.; Zhang, Y.; Wu, S.; Zheng, X. Excavating precursors from the traditional Chinese herb Polygala tenuifolia and Gastrodia elata: Synthesis, anticonvulsant activity evaluation of 3,4,5-trimethoxycinnamic acid (TMCA) ester derivatives. Bioorg. Chem. 2019, 88, 102832. [CrossRef] [PubMed]

26. Dementia Statistics. Alzheimer's Disease International, London, UK. Available online: https://www.alzint.org/about/dementiafacts-figures/dementia-statistics / (accessed on 19 March 2021).

27. Gaugler, J.; James, B.; Johnson, T.; Marin, A.; Weuve, J. 2020 Alzheimer's disease facts and figures. Alzheimer's Dement. 2020, 16, 391-460.

28. Lane, R.M.; Potkin, S.G.; Enz, A. Targeting acetylcholinesterase and butyrylcholinesterase in dementia. Int. J. Neuropsychopharmacol. 2006, 9, 101-124. [CrossRef]

29. Darvesh, S. Butyrylcholinesterase as a diagnostic and therapeutic target for Alzheimer's disease. Curr. Alzheimer Res. 2016, 13, 1173-1177. [CrossRef]

30. Schwarz, S.; Lucas, S.D.; Sommerwerk, S.; Csuk, R. Amino derivatives of glycyrrhetinic acid as potential inhibitors of cholinesterases. Bioorg. Med. Chem. 2014, 52, 3370-3378. [CrossRef]

31. Ibach, B.; Haen, E. Acetylcholinesterase inhibition in Alzheimer's disease. Curr. Pharm. Des. 2014, 10, 231-251. [CrossRef] [PubMed]

32. Colovic, M.B.; Krstic, D.Z.; Lazarevic-Pasti, T.D.; Bondzic, A.M.; Vasic, V.M. Acetylcholinesterase inhibitors: Pharmacology and toxicology. Curr. Neuropharmacol. 2013, 11, 315-335. [CrossRef]

33. Moss, D.E. Improving anti-neurodegenerative benefits of acetylcholinesterase inhibitors in Alzheimer's disease: Are irreversible inhibitors the future? Int. J. Mol. Sci. 2020, 21, 3438. [CrossRef] [PubMed]

34. Darvesh, S.; Hopkins, D.A.; Geula, C. Neurobiology of butyrylcholinesterase. Nat. Rev. Neurosci. 2003, 4, 131-138. [CrossRef] [PubMed]

35. Mesulam, M.; Guillozet, A.; Shaw, P.; Quinn, B. Widely spread butyrylcholinesterase can hydrolyze acetylcholine in the normal and Alzheimer brain. Neurobiol. Dis. 2002, 9, 88-93. [CrossRef]

36. Giacobini, E. Cholinesterase inhibitors: New roles and therapeutic alternatives. Pharmacol. Res. 2004, 50, 433-440. [CrossRef] [PubMed]

37. Cherif, O.; Allouche, F.; Chabchoub, F.; Chioua, M.; Soriano, E.; Yanez, M.; Cacabelos, R.; Romero, A.; Lopez, M.G.; Contelles, J.M. Isoxazolotacrines as nontoxic and selective butyrylcholinesterase inhibitors for Alzheimer's disease. Future Med. Chem. 2014, 6, 1883-1891. [CrossRef]

38. Brus, B.; Kosak, U.; Turk, S.; Pislar, A.; Coquelle, N.; Kos, J.; Stojan, J.; Colletier, J.P.; Gobec, S. Discovery, biological evaluation, and crystal structure of a novel nanomolar selective butyrylcholinesterase inhibitor. J. Med. Chem. 2014, 57, 8167-8179. [CrossRef]

39. Zeb, A.; Hameed, A.; Khan, L.; Khan, I.; Dalvandi, K.; Choudhary, M.I.; Basha, F.Z. Quinoxaline derivatives: Novel and selective butyrylcholinesterase inhibitors. Med. Chem. 2014, 10, 724-729. [CrossRef]

40. Nordberg, A.; Ballard, C.; Bullock, R.; Darreh-Shori, T.; Somogyi, M. A review of butyrylcholinesterase as a therapeutic target in the treatment of Alzheimer's disease. Prim. Care Companion CNS Disord. 2013, 15, PCC.12r01412. [CrossRef]

41. Kandiah, N.; Pai, M.C.; Senanarong, V.; Looi, I.; Ampil, E.; Park, K.W.; Karanam, A.K.; Christopher, S. Rivastigmine: The advantages of dual inhibition of acetylcholinesterase and butyrylcholinesterase and its role in subcortical vascular dementia and Parkinson's disease dementia. Clin. Interv. Aging. 2017, 12, 697-707. [CrossRef]

42. Dighe, S.N.; Tippana, M.; van Akker, S.; Collet, T.A. Structure-based scaffold repurposing toward the discovery of novel cholinesterase inhibitors. ACS Omega 2020, 5, 30971-30979. [CrossRef] 
43. Pospisilova, S.; Kos, J.; Michnova, H.; Kapustikova, I.; Strharsky, T.; Oravec, M.; Moricz, A.M.; Bakonyi, J.; Kauerova, T.; Kollar, P.; et al. Synthesis and spectrum of biological activities of novel $N$-arylcinnamamides. Int. J. Mol. Sci. 2018, 19, 2318. [CrossRef] [PubMed]

44. Hosek, J.; Kos, J.; Strharsky, T.; Cerna, L.; Starha, P.; Vanco, J.; Travnicek, Z.; Devinsky, F.; Jampilek, J. Investigation of antiinflammatory potential of n-arylcinnamamide derivatives. Molecules 2019, 24, 4531. [CrossRef] [PubMed]

45. Kos, J.; Bak, A.; Kozik, V.; Jankech, T.; Strharsky, T.; Swietlicka, A.; Michnova, H.; Hosek, J.; Smolinski, A.; Oravec, M.; et al. Biological activities and ADMET-related properties of novel set of cinnamanilides. Molecules 2020, 25, 4121. [CrossRef]

46. Michnova, H.; Strharsky, T.; Kos, J.; Cizek, A.; Jampilek, J. Antistaphylococcal activity of polychlorinated N-arylcinnamamides. In Proceedings of the 6th International Electronic Conference on Medicinal Chemistry (ECMC-6), Online, 1-30 November 2020; p. 7403. Available online: https://sciforum.net/manuscripts/7403/slides.pdf (accessed on 18 March 2021).

47. Michnova, H.; Strharsky, T.; Pospisilova, S.; Kos, J.; Cizek, A.; Jampilek, J. Anti-infective activity of selected trifluoromethylsubstituted N-Arylcinnamamides. In Proceedings of the 6th International Electronic Conference on Medicinal Chemistry (ECMC-6), Online, 1-30 November 2020; p. 7404. Available online: https:/ / sciforum.net/manuscripts/7404/slides.pdf (accessed on 18 March 2021).

48. Kim, S.; Kim, Y.; Kong, Y.; Kim, H.; Kang, J. Synthesis and in vitro biological activity of retinyl polyhydroxybenzoates, novel hybrid retinoid derivatives. Bioorg. Med. Chem. Lett. 2009, 19, 508-512. [CrossRef] [PubMed]

49. Ellman, G.L.; Courtney, K.D.; Andres, V.; Featherstone, R.M. A new and rapid colorimetric determination of acetylcholinesterase activity. Biochem. Pharmacol. 1961, 7, 88-95. [CrossRef]

50. Ou, S.; Kwok, K.C.; Wang, Y.; Bao, H. An improved method to determine SH and -S-S- group content in soymilk protein. Food Chem. 2004, 88, 317-320. [CrossRef]

51. Sinko, G.; Calic, M.; Bosak, A.; Kovarik, Z. Limitation of the Ellman method: Cholinesterase activity measurement in the presence of oximes. Anal. Biochem. 2007, 370, 223-227. [CrossRef] [PubMed]

52. Zdrazilova, P.; Stepankova, S.; Komers, K.; Ventura, K.; Cegan, A. Half-inhibition concentrations of new cholinesterase inhibitors. Z. Nat. C 2004, 59, 293-296.

53. Kos, J.; Kozik, V.; Pindjakova, D.; Jankech, T.; Smolinski, A.; Stepankova, S.; Hosek, J.; Oravec, M.; Jampilek, J.; Bak, A. Synthesis and hybrid SAR property modeling of novel cholinesterase inhibitors. Int. J. Mol. Sci. 2021, 22, 3444. [CrossRef]

54. Lineweaver, H.; Burk, D. The determination of enzyme dissociation constants. J. Am. Chem. Soc. 1934, 56, 658-666. [CrossRef]

55. Kielczewska, U.; Jorda, R.; Gonzalez, G.; Morzycki, J.W.; Ajani, H.; Svrckova, K.; Stepankova, S.; Wojtkielewicz, A. The synthesis and cholinesterase inhibitory activities of solasodine analogues with seven-membered F ring. J. Steroid Biochem. Mol. Biol. 2021, 205, 105776. [CrossRef] [PubMed]

56. Pejchal, V.; Stepankova, S.; Padelkova, Z.; Imramovsky, A.; Jampilek, J. 1,3-Substituted imidazolidine-2,4,5-triones: Synthesis and inhibition of cholinergic enzymes. Molecules 2011, 16, 7565-7582. [CrossRef] [PubMed]

57. Pauk, K.; Zadrazilova, I.; Imramovsky, A.; Vinsova, J.; Pokorna, M.; Masarikova, M.; Cizek, A.; Jampilek, J. New derivatives of salicylamides: Preparation and antimicrobial activity against various bacterial species. Bioorg. Med. Chem. 2013, 21, 6574-6581. [CrossRef]

58. Kos, J.; Nevin, E.; Soral, M.; Kushkevych, I.; Gonec, T.; Bobal, P.; Kollar, P.; Coffey, A.; O’Mahony, J.; Liptaj, T.; et al. Synthesis and antimycobacterial properties of ring-substituted 6-hydroxynaphthalene-2-carboxanilides. Bioorg. Med. Chem. 2015, 23, 2035-2043. [CrossRef] [PubMed]

59. Kos, J.; Zadrazilova, I.; Nevin, E.; Soral, M.; Gonec, T.; Kollar, P.; Oravec, M.; Coffey, A.; O’Mahony, J.; Liptaj, T.; et al. Ringsubstituted 8-hydroxyquinoline-2-carboxanilides as potential antimycobacterial agents. Bioorg. Med. Chem. 2015, 23, $4188-4196$. [CrossRef] [PubMed]

60. Kapustikova, I.; Bak, A.; Gonec, T.; Kos, J.; Kozik, V.; Jampilek, J. Investigation of hydro-lipophilic properties of Nalkoxyphenylhydroxynaphthalenecarboxamides. Molecules 2018, 23, 1635. [CrossRef] [PubMed]

61. Kos, J.; Zadrazilova, I.; Pesko, M.; Keltosova, S.; Tengler, J.; Gonec, T.; Bobal, P.; Kauerova, T.; Oravec, M.; Kollar, P.; et al. Antibacterial and herbicidal activity of ring-substituted 3-hydroxynaphthalene-2-carboxanilides. Molecules 2013, 18, $7977-7997$. [CrossRef]

62. DrugBank-Rivastigmine. Available online: https://go.drugbank.com/drugs/DB00989 (accessed on 7 May 2021).

63. DrugBank-Galantamine. Available online: https://go.drugbank.com/drugs/DB00674 (accessed on 7 May 2021).

64. Imramovsky, A.; Stepankova, S.; Vanco, J.; Pauk, K.; Monreal-Ferriz, J.; Vinsova, J.; Jampilek, J. Acetylcholinesterase-inhibiting activity of salicylanilide $N$-alkylcarbamates and their molecular docking. Molecules 2012, 17, 10142-10158. [CrossRef]

65. Imramovsky, A.; Pejchal, V.; Stepankova, S.; Vorcakova, K.; Jampilek, J.; Vanco, J.; Simunek, P.; Kralovec, K.; Bruckova, L.; Mandikova, J.; et al. Synthesis and in vitro evaluation of new derivatives of 2-substituted-6-fluorobenzo[d]thiazoles as cholinesterase inhibitors. Bioorg. Med. Chem. 2013, 21, 1735-1748. [CrossRef]

66. Pizova, H.; Havelkova, M.; Stepankova, S.; Bak, A.; Kauerova, T.; Kozik, V.; Oravec, M.; Imramovsky, A.; Kollar, P.; Bobal, P.; et al. Prolin-based carbamates as cholinesterase inhibitors. Molecules 2017, 22, 1969. [CrossRef]

67. Bak, A.; Kozik, V.; Kozakiewicz, D.; Gajcy, K.; Strub, D.J.; Swietlicka, A.; Stepankova, S.; Imramovsky, A.; Polanski, J.; Smolinski, A.; et al. Novel benzene-based carbamates for AChE/BChE inhibition: Synthesis and ligand/structure-oriented SAR study. Int. J. Mol. Sci. 2019, 20, 1524. [CrossRef] [PubMed] 
68. Bak, A.; Pizova, H.; Kozik, V.; Vorcakova, K.; Kos, J.; Treml, J.; Odehnalova, K.; Oravec, M.; Imramovsky, A.; Bobal, P.; et al. SAR-mediated similarity assessment of property profile for new silicon-based AChE/BChE inhibitors. Int. J. Mol. Sci. 2019, 20, 5385. [CrossRef] [PubMed] 